\title{
Brain Correlates of Language Learning: The Neuronal Dissociation of Rule-Based versus Similarity-Based Learning
}

\author{
Bertram Opitz ${ }^{1}$ and Angela D. Friederici ${ }^{2}$ \\ ${ }^{1}$ Experimental Neuropsychology Unit, Saarland University, 66041 Saarbrücken, Germany, and 2Department of Neuropsychology, Max Planck Institute for \\ Human Cognitive and Brain Sciences, 04303 Leipzig, Germany
}

\begin{abstract}
Language learning is one of the mysteries of human cognition. One of the crucial questions is the following: Does acquisition of grammatical knowledge depend primarily on abstract rules or on item-specific information? Although there is evidence that both mechanisms contribute to language acquisition, their relative importance during the process of language learning is unknown. Using an artificial grammar paradigm, we show by means of functional magnetic resonance imaging that the brain dissociates the two mechanisms: the left anterior hippocampus supports similarity-based learning, whereas the left ventral premotor cortex is selectively engaged by abstract rule processing. Moreover, data analysis over time on learning suggests that similarity-based learning plays a nonobligatory role during the initial phase, and that rule-based abstraction plays a crucial role during later learning.
\end{abstract}

Key words: hippocampus; rule learning; grammar; premotor cortex; fMRI; similarity

\section{Introduction}

The processes by which humans learn a language have gained considerable interest over the past years (Hauser et al., 2002). Based on the idea that language is so complex (Chomsky, 1965), the acquisition of grammatical knowledge has been widely assumed to involve structural rules. These so-called phrase structure rules (PSRs) determine how words are combined into phrases and sentences. In contrast, another type of grammar, called finite-state grammar (FSG), is specified by transition probabilities between elements. For the processing of FSGs implemented in artificial grammar-learning tasks (Reber, 1967), a mechanism has been proposed that considers the similarity to exemplars presented during training (Seger, 1994; Shanks, 1995). It has been argued that this form of learning may well explain the acquisition of FSGs. In a number of experiments, Vokey and Brooks (1992) demonstrated that similarity-based learning leads to transfer of grammatical knowledge to a new letter-set in a way similar to rule-based learning. Reconciling the divergent results, it has been proposed that performance in such tasks depends on both item similarity and rule knowledge (Brooks and Vokey, 1991; Knowlton and Squire, 1996). Such proposals necessarily raise the question of how such resources actually interact and which brain systems mediate them.

It is commonly agreed that rule-based learning of natural grammars is mediated by the left prefrontal cortex, especially Broca's area (Musso et al., 2003; Opitz and Friederici, 2003), because it is the rule-based processing center of acquired syntax

Received March 18, 2004; revised July 23, 2004; accepted July 23, 2004.

This work was supported by grants from the German Research Foundation (Deutsche Forschungsgemeinschaft) Research Group F0R-448.

Correspondence should be addressed to Dr. Bertram Opitz, Experimental Neuropsychology Unit, Saarland University, P.0. Box 1511 50, 66041 Saarbrücken, Germany. E-mail: b.opitz@mx.uni-saarland.de.

DOI:10.1523/JNEUROSCI.2220-04.2004

Copyright $\odot 2004$ Society for Neuroscience $\quad$ 0270-6474/04/248436-05\$15.00/0
(Ullman et al., 1997; Indefrey et al., 2001). With respect to similarity-based learning mechanisms, there is less converging evidence. Recent studies that use functional magnetic resonance imaging (fMRI) have proposed the role of the left hippocampus in similarity-based learning (Strange et al., 2001; Opitz and Friederici, 2003). In the study by Strange et al. (2001), subjects made grammaticality judgments to letter strings for which the governing rule and the letters that comprised the exemplars were periodically changed. Activity in the left hippocampus was modulated by changes of exemplars, but not by changes of grammatical rules. However, FSG learning based on exemplar-specific information has been shown to be intact in patients suffering from global amnesia and thus to be independent of the hippocampal system (Knowlton and Squire, 1996).

To investigate the neural basis of both learning mechanisms, we created an artificial grammar consisting of PSRs and a small vocabulary. Once learned, participants were presented with language input, which was systematically changed in terms of either the phrase structure rules or one feature of a particular word category. This design of this fMRI study allowed us to evaluate modulations of brain activity as a function of the two types of changes while keeping everything else constant. If the hippocampal formation plays a prominent role in similarity-based learning, it should be activated by changes of words in a particular category. In contrast, changes of the underlying phrase structure rules might modulate activation in the left prefrontal cortex.

\section{Materials and Methods}

Subjects. A total of 21 right-handed, monolingual participants (12 male; mean age, 25 years) with no history of neurological or psychiatric disorder volunteered in this study. All of the subjects were trained on the miniature artificial grammar system BROCANTO (Friederici et al., 2002; Opitz and Friederici, 2003) $\sim 48 \mathrm{hr}$ before the experiment. The training procedure was identical with that described in Opitz and Friederici (2003). Participants were given observation training on a list of 10 sen- 


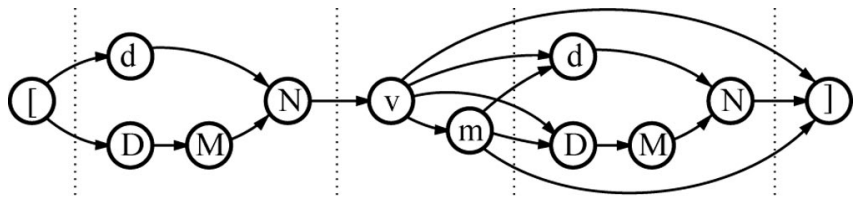

syntactic structures:

\section{$S$ : NP VP NP VP NP}

NP : d N $\mathrm{D} \mathrm{M} \mathrm{N}$

VP : V $\mathrm{V}$ m word categories:

$\mathrm{N}:$ gum, plox, tok, trul

$\mathrm{V}$ : glif, pel, prez, rix

$\mathrm{M}$ : böke

$\mathrm{m}:$ rüfi

d : aaf D : aak
Figure 1. Schematic representation of the artificial grammar of BROCANTO. Nodes in the top panel specify word classes (nouns, verbs, etc.), whereas arrows denote valid transitions between nodes. A correct sentence is formed by a transition from beginning ([) to end (]). The bottom panel depicts the rules according to which valid phrases are formed. Thus, a sentence $(S)$ consists of a noun phrase (NP) and a verb phrase (VP). A NP in turn is either the sequence $\mathrm{dN}$ or DMN, where $\mathrm{N}$ is one of the possible noun choices. Word categories: $N$, noun; $v$, verb; $M$, adjective; $m$, adverb; $d$, determiner in NP; $D$, determiner in modified NP (i.e., NP with adjective).

tences and were instructed to extract the underlying grammatical rules. After training, participants were asked to judge the correctness of a new set of sentences according to the grammar they learned. This procedure was repeated several times with different sentences. Training ended when either $85 \%$ correct grammaticality responses in 30 successive sentences were made, or when a total of 150 sentences were accomplished. The average learning success rate was $80 \%$ and ranged from 63 to $99 \%$.

Stimuli. Thirty sentences were formulated representing a subjectverb-[object] structure (Fig. 1), according to the grammar system BROCANTO. BROCANTO is based on the universal principles of natural languages (i.e., it consists of different syntactic word categories and defined phrase structure rules). Another 30 sentences contained a severe syntactic violation: an agreement violation, a word category repetition, or a phrase structure violation (Opitz and Friederici, 2003). See Table 1 for examples.

Procedure. Subjects were repeatedly presented with series of three blocks in the same order: a learning block, a test block, and a sensorimotor control block. During learning, participants viewed 10 correct sentences on a computer monitor and were instructed to extract the underlying grammatical rules. During test blocks, participants were presented with 10 sentences, one-half of which were ungrammatical. The participants' task was a grammaticality judgment on each sentence presented. Feedback was given for each response. The grammatically correct sentences used during the test phase were different from those used during learning to reduce the likelihood of reliance on recall of correct sentences. To control for unspecific changes over time, a control task was introduced that consisted of serial visual presentation of either of two pseudowords (BRAD and DABA) that did not belong to the grammar of BROCANTO. Because a forced-choice button press relating to which pseudoword had been presented was required, the control task was matched for stimulus properties (pseudowords) and response requirements (forced-choice button press).

After three series of initial exposure to the original grammar system of BROCANTO were accomplished, systematic changes to the grammar were incorporated. Subjects were assigned to two groups for which one parameter of the grammar was varied in the test cycle. A between-group design was necessary to investigate the change of the two crucial parameters after initial learning. For one-half of the subjects, the superficial similarity was changed by allowing the noun to vary in position in the noun phrases without violating the underlying phrase structure rules of BROCANTO. This WORD condition meant that nouns that were presented only in the sentence-initial-noun phrase during the early training would now also appear in the sentence-final-noun phrase. The WORD condition resulted in two positional changes (Table 1). For example, the noun "plox" was presented only in subject position (as in 1) during training, but appeared also in object position during test (as in 2).

For the other one-half of the participants, a new phrase structure rule (RULE condition) was incorporated into BROCANTO. The added rule concerned the modifier-noun relationship. Although the original grammar of BROCANTO always placed the modifier before the noun [as in (1), "böke gum"], the test version allowed the modifier to follow the noun to which it refers as well [as in (3), "gum böke"]. Note that in terms of "editing distance" or the changes needed to make the training and test sentences identical, both WORD and RULE conditions resulted in two positional changes.

Data acquisition. Imaging was performed on a 3T-Bruker Medspec 30/100 system using a standard birdcage head coil. In a separate session before the experiment, high-resolution three-dimensional structural images were collected using an MDEFT sequence. Functional images sensitive to BOLD contrast were acquired with a gradient-echo echo-planar imaging sequence (echo time, $30 \mathrm{msec}$; flip angle $90^{\circ}$; repetition time, 1 $\mathrm{sec}$ ). An acquisition volume consisted of 12 slices ( $5 \mathrm{~mm}$ thickness, $2 \mathrm{~mm}$ gap) parallel to the plane intersecting the anterior and posterior commissures with an in-plane resolution of $3 \mathrm{~mm}^{2}$. Slices were positioned individually to cover the medial temporal lobe up to the superior frontal sulcus. A total of 2670 functional volumes per participant were acquired.

Data analysis. Data were analyzed analogous to our previous study (Opitz and Friederici, 2003) using the general linear model as implemented in the software package LIPSIA (Lohmann et al., 2001). The data were smoothed spatially with an isotropic $6 \mathrm{~mm}$ full-width at halfmaximum Gaussian kernel, and the global means were normalized by proportional scaling. For each subject, neural activity in each block was modeled by convolving a stimulus function representing this block with a canonical hemodynamic response function (Friston et al., 1998). The resulting subject-specific time series of each experimental block were weighted with that subject's behavioral performance measure. This parameter function was derived from each individual's behavioral performance (percentage of correct responses in each block) by fitting a logarithmic function to the five time bins of the learning curve separately for each condition. The two original condition-specific model functions (WORD/RULE conditions vs the sensorimotor control condition) were multiplied by the learning function. This led to additional regressors reflecting learning-related changes in the WORD/RULE conditions relative to the almost enduring activity in the sensorimotor control condition. Linear contrasts of the parameter estimates for each regressor were calculated and entered into a second-level analysis treating subjects as a random effect using a one-sample $t$ test against a contrast value of zero at each voxel. Activations were considered significant when comprised of 10 or more contiguous voxels surviving a threshold of $p<0.0001$, uncorrected.

\section{Results}

The performance in terms of percentage correct and reaction times of both groups are depicted in Figure 2. As apparent from Figure 2, performance during the initial three blocks did not differ between both groups (both $F$ values, $<1$ ), indicating that both groups had the same level of competence in the original grammar of BROCANTO. The introduction of the RULE change led to a significant decrease in grammaticality judgment performance $\left(F_{(1,10)}=5.13 ; p<0.025\right.$; one-tailed $)$ and an increase in reaction time $\left(F_{(1,10)}=149.68 ; p<0.0001\right)$ immediately after the change with subsequent learning of the new rule. In contrast, in the WORD condition, performance improved throughout the entire session (Fig. 2A), although reaction times slightly increased after the change of superficial word position $\left(F_{(1,9)}=8.1\right.$; $p<0.05)$. There was no significant difference in performance and reaction times between the groups as confirmed by an ANOVA contrasting the groups' performance across blocks (performance, $F_{(1,19)}=0.66 ; p>0.42$; reaction time, $F_{(1,19)}=3.4 ; p>$ 
0.1). However, there was a significant block-by-group interaction for performance $\left(F_{(4,76)}=3.77 ; p<0.01\right)$, pointing to differential processing of WORD and RULE changes.

Concerning the fMRI data, a randomeffects model was used to identify the activation pattern specific to the acquisition of the changed version of BROCANTO. This was reflected by the interaction between the condition (WORD/RULE condition vs asensorimotor control condition) and the individual accuracy of performance. Our contrasts tested for greater learning-related changes in the BOLD signal in the learning blocks versus sensorimotor control blocks separate for the WORD and RULE conditions, thereby allowing us to compare the involvement of particular brain structures in rule-based and similarity-based learning (see Materials and Methods). Increased proficiency in grammar use as indicated by the learning curve (Fig. 2A) resulted in a significant modulation of left anterior hippocampal activity (coordinates, $-21,-17,-17$ ) (Talairach and Tournoux, 1988) in the WORD condition only (Fig. 3, left panel). Interestingly, for the WORD condition, we observed a transient initial increase between the first three blocks (original grammar) and the fourth block with the change followed by a gradual decrease immediately after the change in superficial similarity.

In contrast, the RULE condition indicated a gradual increase of activity in the left ventral premotor cortex (Talairach coordinates, $-43,0,17$ ) (Fig. 3). Crucially, this increase was caused by the introduction of a new phrase structure rule in the fourth learning block (Fig. 3, right panel, arrow).

To investigate the differential processing of WORD and RULE changes further, we contrasted the signal change within the left hippocampus and the left ventral premotor cortex (vPMC) directly between conditions. A repeated-measure ANOVA treating condition (RULE vs WORD) as a between-subjects factor and block ( 5 time bins equivalent to the learning curve) as a withinsubject factor revealed a significant block-by-condition interaction for both areas (hippocampus, $F_{(4,76)}=2.91 ; p<0.05$; vPMC, $\left.F_{(4,76)}=2.59 ; p<0.05\right)$. These differential activation patterns emerged only after the WORD and RULE condition changes were introduced. That is, that the brain activity during the first three blocks (comprised of the original version of BROCANTO) did not differ between groups ( $F$ values for both regions, $<1$ ).

\section{Discussion}

The present experiment set out to investigate the neural correlates of similarity-based and rule-based acquisition. We did this by using an artificial grammar system, having participants learn it in its original form, and then introducing systematic changes. Brain activity was scanned during the learning of the novel forms of the grammar. Clear dissociations were observed between both types of learning in grammaticality judgment performance and in brain activation.

Behaviorally, changes in word position without any phrase structure rule change (WORD condition) did not affect learning performance, whereas the introduction of a new phrase structure rule led to decreased performance. Thus, the WORD condition showed that items adhering to learned grammatical rules (i.e., nouns and their possible positions in the phrase structure) were judged to be grammatical, although they differed superficially from the learned exemplars (i.e., their actual positions in the sentences during training). These behavioral results suggest that compliance with grammatical rules might be the dominant factor guiding grammatical classification judgment (Knowlton and Squire, 1996).

Although the WORD condition exhibited no effects in terms of grammaticality judgment performance, a clear modulation of brain activity was found in the left anterior hippocampus. The initial increase of activation reflects the engagement of the hippocampal system when confronted with changes in word position, whereas the following decrease indicates that less hippocampal resources are needed when learning proceeds. It has been argued independently that similarity-based learning, which operates on perceptual characteristics of each word in a sentence, can be assumed as a specific form of relational processing (Gomez and Gerken, 2000). Relational processing, in turn, has been proposed to be a function of the hippocampal system (Cohen et al., 1999; Davachi and Wagner, 2002). Within this framework, the hippocampal activity observed here might be interpreted to reflect the resources needed to recall learned relationships between words and their possible positions, and these reduce as learners become more proficient. However, with respect to the present grammatical system, this means that words have to be bound to their specific syntactic categories (e.g., noun) and thereby their possible positions (e.g., subject-object position) within a sentence. This means that the introduction of a new relationship of words within a sentence would lead to enhanced activity of the left hippocampal formation because of increased relational processing demands (Cohen et al., 1999; Schacter and Wagner, 1999). Repeated occurrence of a particular relationship facilitates the binding of a word to its syntactic category and thereby its role in the sentence. Therefore, less left anterior hip- 

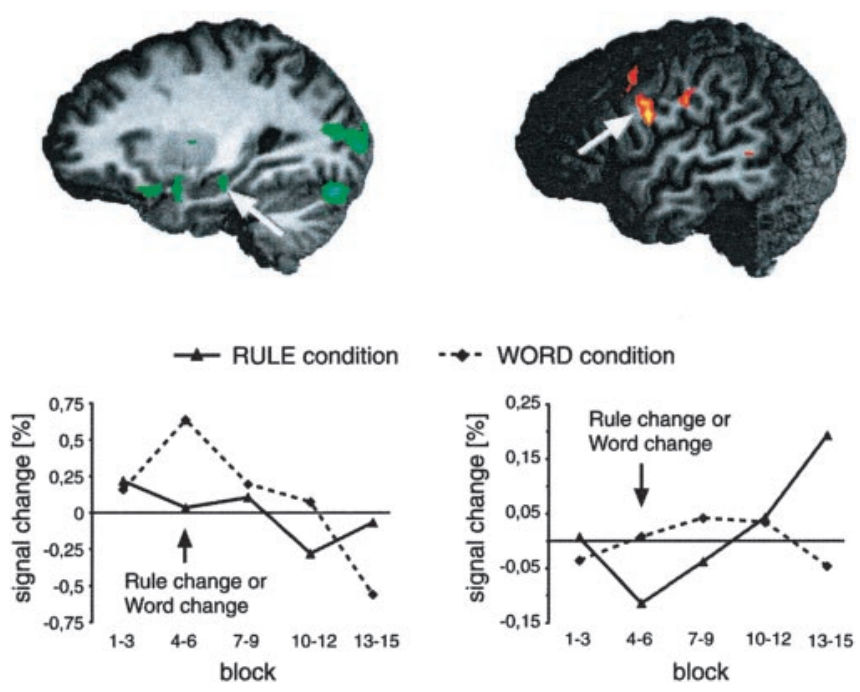

Figure 3. Brain regions in which a significant condition-by-time interaction was observed. The sagittal sections at $x=-26 \mathrm{~mm}$ (left panel) and $x=-48 \mathrm{~mm}$ exhibit brain areas with changes of activity during learning relative to recurring control. Decreased activity in the left anterior hippocampus was noted for similarity-based learning only (top left panel, indicated by arrow). Regions demonstrating increased activity during learning included the left ventral premotor cortex and was predominant for rule-based learning (top right panel). Time courses of brain activity in the hippocampus (bottom left) and the premotor cortex (bottom right) illustrate this differential effect.

pocampal activity is observed as a function of learning a new word position without a rule change in a language-like artificial grammar. This is consistent with the view that the left hippocampal system mediates similarity-based learning, which dominates the initial phase of the acquisition of a language-like grammar (Opitz and Friederici, 2003). The present results receive additional support from recent imaging studies that demonstrate decreased hippocampal activity during the time course of associative learning (Zeineh et al., 2003) and of probabilistic cue-outcome learning (Poldrack et al., 2001).

Despite this converging fMRI evidence, there is some apparent inconsistency with previous neuropsychological findings. Knowlton and Squire (1996) reported that similarity between items influenced the grammaticality judgment of amnesic patients and healthy controls in the same way, suggesting that similarity-based learning does not necessarily depend on the hippocampal formation. There are several possible explanations for the inconsistency of the patient data with the present data. The patient effects may stem from the heterogeneity of the patient sample (most of the patients suffer from diencephalic damage). Alternatively, even a small amount of hippocampal functioning is sufficient to support similarity-based learning of a language-like artificial grammar. Another explanation for these contradictory results, however, could be derived from the way superficial similarity was manipulated in the different studies. In the study by Knowlton and Squire (1996), nongrammatical items could either contain impermissible chunks (themselves violating the grammatical rules) or contain permissible chunks that appear in impermissible locations. The latter type was more readily endorsed as grammatical than the impermissible chunks whose ungrammaticality was easily detected (Gomez and Schvanenfeldt, 1994). In the present study, the changes of word position resemble the harder-to-detect, grammatically permissible chunks in different locations. Thus, it is conceivable that these word position changes engage the hippocampus system more than the detection of impermissible chunks.
The RULE condition, in contrast, triggered an increased engagement of the left vPMC. This is in agreement with recent results demonstrating the involvement of this brain region in the acquisition of grammatical rules (Tettamanti et al., 2002). The focus of the present activation is somewhat more posterior to previously observed activations in correlation with grammatical rule processing (Musso et al., 2003; Opitz and Friederici, 2003), but the study by Tettamanti et al. (2002) reported two activation foci for the comparison between grammatical and nongrammatical rules, a more anterior activation in Brodman area (BA) 44/45 $(-44,14,4)$ and a more posterior activation in BA $6(-50,-4$, 28). Activations in BA 44 have been reported in a number of studies on syntactic transformations in natural languages (Just et al., 1996; Stromswold et al., 1996; Embick et al., 2000; Fiebach et al., 2001; Ben-Shachar et al., 2003), whereas the left premotor cortex was shown to be activated during detection of local ungrammaticalities (Friederici et al., 2003). The new rule introduced in the present study is a local phrase structure one, because it concerns a change in modifier placement in the noun phrase; therefore, the activation in the left vPMC may be related to the local character of rule change. This suggests that the activation observed in the left vPMC is not restricted to the detection of local ungrammaticalities, but more generally supports the processing of local structures, be it during learning or ungrammaticality detection.

Together, the current findings agree with previous studies demonstrating that changes in superficial features of a novel grammar modulate activation in the left anterior hippocampus, whereas changes in abstract rules selectively engage prefrontal cortices. First, these results point to a dissociation between similarity-based learning and rule abstraction during language acquisition. Second, they suggest that similarity-based learning plays a nonobligatory role during initial learning, whereas rulebased abstraction plays a crucial role during later learning. This is in agreement with a recent model of the functional architecture of first and second language learning (Ullman, 2001) proposing that language processing depends on temporal lobe structures as well as left inferior frontal and basal-ganglia structures. Crucially, based on previous event-related potential (ERP) studies that have consistently shown an enhanced negativity over posterior brain areas for the less proficient second language processing of syntactic violations (Weber-Fox and Neville, 1996; Hahne and Friederici, 2001), this model posits prevailing involvement of the temporal lobes in grammar processing in the second language. In contrast, high proficient adult second language learners exhibited an early frontal ERP effect of syntactic processing, typically observed for native speakers (Friederici et al., 2002). This is consistent with the present results suggesting initial involvement of the left hippocampus, with a shift to left inferior frontal activity associated with the proficient use of a language-like artificial grammar.

\section{References}

Ben-Shachar M, Hendler T, Kahn I, Ben-Bashat D, Grodzinsky Y (2003) The neural reality of syntactic transformations: evidence from fMRI. Psychol Sci 14:433-440.

Brooks RL, Vokey RJ (1991) Abstract analogies and abstracted grammars: comments on Reber (1989) and Mathews et al. (1989). J Exp Psychol Learn Mem Cogn 120:316-323.

Chomsky N (1965) Aspects of the theory of syntax. Cambridge, MA: MIT.

Cohen NJ, Ryan E, Hunt C, Romine L, Wszalek T, Nash C (1999) Hippocampal system and declarative (relational) memory: summarizing the data from functional neuroimaging studies. Hippocampus 9:83-98.

Davachi L, Wagner AD (2002) Hippocampal contributions to episodic en- 
coding: insights from relational and item-based learning. J Neurophysiol 88:982-990.

Embick D, Marantz A, Miyashita Y, O’Neil W, Sakai KL (2000) A syntactic specialization for Broca's area. Proc Natl Acad Sci USA 97:6150-6154.

Fiebach CJ, Schlesewsky M, Friederici AD (2001) Syntactic working memory and the establishment of filler-gap dependencies: insights from ERPs and fMRI. J Psychol Res 30:321-338.

Friederici AD, Steinhauer K, Pfeifer E (2002) Brain signatures of artificial language processing: evidence challenging the "critical period" hypothesis. Proc Natl Acad Sci USA 99:529-534.

Friederici AD, Rüschemeyer S-A, Hahne A, Fiebach CJ (2003) The role of left inferior frontal and superior temporal cortex in sentence comprehension: localizing syntactic and semantic processes. Cereb Cortex 13:170-177.

Friston KJ, Fletcher P, Josephs O, Holmes AP, Rugg MD, Turner R (1998) Event-related fMRI: characterizing differential responses. NeuroImage $7: 30-40$.

Gomez RL, Gerken LA (2000) Infant artificial language learning and acquisition learning. Trends Cogn Sci 4:178-186.

Gomez RL, Schvanenfeldt RW (1994) What is learned from artificial grammars? Transfer tests of simple association. J Exp Psychol Learn Mem Cogn 20:396-410.

Hahne A, Friederici AD (2001) Processing a second language: late learners' comprehension mechanisms as revealed by event-related brain potentials. Bilingualism Lang Cogn 4:123-141.

Hauser MD, Chomsky N, Fitch WT (2002) The faculty of language: what is it, who has it, and how did it evolve? Science 298:1569-1579.

Indefrey P, Hagoort P, Herzog H, Seitz RJ, Brown CM (2001) Syntactic processing in left prefrontal cortex is independent of lexical meaning. NeuroImage 14:546-555.

Just MA, Carpenter PA, Keller TA, Eddy WF, Thulborn KR (1996) Brain activation modulated by sentence comprehension. Science 274:114-116.

Knowlton BJ, Squire LR (1996) Artificial grammar learning depends on implicit acquisition of both abstract and exemplar-specific information. J Exp Psychol Learn Mem Cogn 22:169-181.

Lohmann G, Mueller K, Bosch V, Mentzel H, Hessler S, Chen L, Zysset S, von Cramon DY (2001) A new software system for the evaluation of functional magnetic resonance images of the human brain. Comput Med Imaging Graph 25:449-457.
Musso M, Moro A, Glauche V, Rijntjes M, Reichenbach J, Buchel C, Weiller C (2003) Broca's area and the language instinct. Nat Neurosci 6:774-781.

Opitz B, Friederici AD (2003) Interactions of the hippocampal system and the prefrontal cortex in learning language-like rules. NeuroImage 19:1730-1737.

Poldrack RA, Clark J, Paré-Blagoev EJ, Shohamy D, Creso Moyano J, Myers C, Gluck MA (2001) Interactive memory systems in the human brain. Nature 414:546-550.

Reber AS (1967) Implicit learning of artificial grammars. J Verbal Learn Verbal Behav 6:855-863.

Schacter DL, Wagner AD (1999) Remembrance of things past. Science 285:1503-1504.

Seger CA (1994) Implicit learning. Psychol Bull 115:163-196.

Shanks DR (1995) The psychology of associative learning. Cambridge, UK: Cambridge UP.

Strange BA, Henson RNA, Friston KJ, Dolan RJ (2001) Anterior prefrontal cortex mediates rule learning in humans. Cereb Cortex 11:1040-1046.

Stromswold K, Caplan D, Alpert N, Rauch S (1996) Localization of syntactic comprehension by positron emission tomography. Brain Lang 52:452-473.

Talairach J, Tournoux P (1988) Co-planar stereotaxis atlas of the human brain. New York: Thieme.

Tettamanti M, Alkadhi H, Moro A, Perani D, Kollias S, Weniger D (2002) Neural correlates for the acquisition of natural language syntax. NeuroImage 17:700-709.

Ullman MT (2001) The neuronal basis of lexicon and grammar in first and second language: the declarative/procedural model. Bilingualism Lang Cogn 4:105-122.

Ullman MT, Corkin S, Coppola M, Hickok G, Growdon JH, Koroshetz WJ, Pinker SA (1997) A neural dissociation within language - evidence that the mental dictionary is part of declarative memory and that grammatical rules are processed by the procedural system. J Cogn Neurosci 9:266-276.

Vokey JR, Brooks LR (1992) Salience of item knowledge in learning artificial grammar. J Exp Psychol Learn Mem Cogn 20:328-344.

Weber-Fox CM, Neville HJ (1996) Maturational constraints on functional specializations for language processing: ERP and behavioral evidence in bilingual speakers. J Cogn Neurosci 8:231-256.

Zeineh MM, Engel SA, Thompson PM, Bookheimer SY (2003) Dynamics of the hippocampus during encoding and retrieval of face-name pairs. Science 299:577-580. 